\title{
STANDARDIZATION PATHS FOR NGN IMS-BASED ARCHITECTURE
}

Thanks to the rapid development of technology and increased availability of bandwidth, today more than ever, multiple services such as voice, video and data can be integrated and efficiently carried over a single infrastructure based on a packet network. An NGN (Next Generation Network) is able to deploy those services independently from underlying transport-related technologies, giving fixed or mobile users an access to different providers with the adequate QoS and security. In fact, it is the IMS (IP Multimedia Subsystem) inside the NGN that enables and drives efficient converged service offerings. IMS provides the framework for a common service and session control layer standardized on $I P$, with SIP controlling all media sessions. The IMS standardization breaks down all the functions necessary to support multimedia applications and to provide standardized (open) interfaces towards application servers enabling enhanced premium services as well as the interworking with other (legacy) networks such as the PSTN/PLMN (Public Switched Telephone Network/Public Land Mobile Network. The paper is describing the current state of the art in the NGN standardization within the ETSI and related standardization bodies.

\section{Introduction}

The European Telecommunications Standards Institute (ETSI) defines fixed-mobile convergence as being concerned with providing network and service capabilities independently of the access technique. It is concerned with developing converged network capabilities and supporting standards. This does not necessarily imply the physical convergence of networks. These standards may be used to offer a set of consistent services via fixed or mobile access to fixed or mobile, public or private, networks. In other words, fixed-mobile convergence allows users to access a consistent set of services from any fixed or mobile terminal via any compatible access point. An important extension of this principle relates to roaming: users should be able to roam from network to network while using the same consistent set of services throughout those visited networks.

The full evolution to fixed-mobile convergence will be through the NGN (Next Generation Network) path. NGNs promise to be multiservice, multiprotocol, multi-access, IP-based networks: secure, reliable and trusted. The NGN framework is set by the International Telecommunication Union-Telecommunication Standardization Sector (ITU-T) and ETSI (European Telecommunications Standards Institute), especially its Technical Committee TISPAN (TC TISPAN) [1]. Other standardization organizations and fora such as the Internet Engineering Task Force (IETF) [2], 3GPP (Third Generation Partnership Project) [3], 3GPP2, American National Standards Institute (ANSI) [5], CableLabs [6], MultiService Forum (MSF) [7], and Open Mobile Alliance (OMA) [8] are actively involved in defining NGN standards.
In section 2, we provide a brief overview of the ETSI TC TISPAN (ETCI Technical Committee Telecoms \& Internet converged Services \& Protocols for Advanced Networks). In section 3 , the emphasis is on NGN architecture principles and functions. Section 4 deals with the TISPAN NGN Releases and identifies steps in the standardization path towards the full NGN. Finally, conclusions are given in section 5 .

\section{ETSI TC TISPAN}

The Technical Committee TISPAN is the ETSI core competence centre for fixed networks and for migration from switched circuit networks to packet-based networks with an architecture that can serve in both.

TISPAN is responsible for all aspects of standardization for present and future converged networks including the NGN, service aspects, architectural aspects, protocol aspects, QoS (Quality of Service) studies, security related studies, mobility aspects within fixed networks, using existing and emerging technologies. TISPAN is structured as a single Technical Committee, with seven Working Groups to deliver specifications back up to the TISPAN Plenary meetings.

\section{NGN Architecture}

TC TISPAN developed a functional architecture [9] consisting of a number of subsystems and structured in a service layer

\footnotetext{
* Tatiana Kovacikova, Pavol Segec, Michal Bruncko

Department of InfoCom Networks, Faculty of Management Sciences and Informatics, University of Zilina, Slovakia,

E-mail: Tatiana.Kovacikova@fri.uniza.sk
} 
and an IP (Internet Protocol)-based transport layer. This subsystem oriented architecture enables new subsystems to be added over time to cover new demands and service classes. It also provides the ability to import sub-systems defined by other standardization bodies. Each subsystem is specified as a set of functional entities and related interfaces. Fig. 1 shows the overall NGN functional architecture.

The transport layer provides the IP connectivity for NGN users The transport layer comprises a transport control sublayer on top of transport processing functions in the access and core networks. Equivalent functionality in the User Equipment is defined in [9] The transport control sub-layer is further divided into the Network Attachment Subsystem (NASS) and the Resource and Admission Control Subsystem (RACS)

The NASS provides registration at the access level and initializes terminal accessing to NGN services. More specifically, the NASS provides the following functionalities [10]:

- dynamic provision of IP addresses and other terminal configuration parameters;

- authentication taking place at the IP layer, prior or during the address allocation procedure;

- authorization of network access based on user profiles;

- access network configuration based on user profiles;

- location management taking place at the IP layer.

There may be more than one NASS to support multiple access networks.

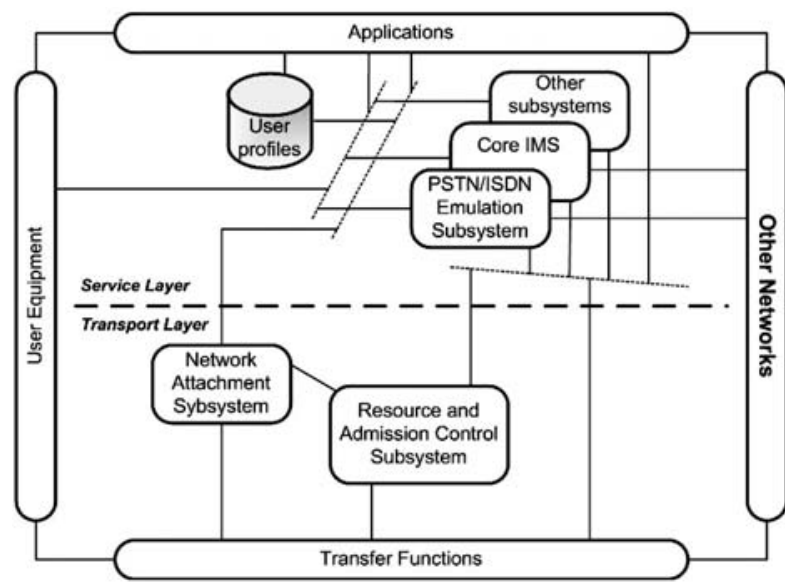

Fig. 1 TISPAN_NGN Overall architecture - Concept of subsystems

RACS [11] is the TISPAN NGN subsystem responsible for the implementation of procedures and mechanisms handling policybased resource reservation and admission control for both unicast and multicast traffic in access networks and core networks. Besides acting as a resource control framework, RACS also includes support for controlling Network Address Translation (NAT) at the edge of networks and assisting in remote NAT traversal. Furthermore RACS also covers aspects related to the setting and modification of traffic policies, end to end quality of service and transport-level charging.

The NGN service layer comprises:

- Core IP Multimedia Subsystem (IMS)

- PSTN/ISDN emulation subsystem (PES) [5]

- IPTV subsystem (The TISPAN architecture also enables supporting IPTV services using the IP Multimedia Subsystem)

- Common components used by several subsystems (User Profile Server Function (UPSF); Subscription Locator Function (SLF); Application Server Function (ASF); Interworking Function (IWF)).

The core network of NGN Release 1 was based upon the IMS (IP Multimedia Subsystem), as defined in 3GPP Release 6 and 3 GPP2 revision A for IP-based multimedia applications. IMS is a framework architecture - a definition of capabilities specified in a set of 3GPP documents that defines components, services and interfaces for NGN. It uses Voice over IP (VoIP) implementation based on a 3GPP standardized implementation of Session Initiation Protocol (SIP), and it runs over the standard Internet Protocol. 3GPP has enhanced the SIP and IP-based protocols (primarily Diameter) to allow for mobility. The TISPAN TC has adopted the IMS and is closely working with 3GPP on any modifications or improvements that may be needed for the NGN [11]. Moreover, the 3GPP IMS has been extended in the TISPAN NGN to support additional access network types, such as XDSL ( $\mathrm{x}$ - Digital Subscriber Line) and WLAN (Wireless Local Area Network).

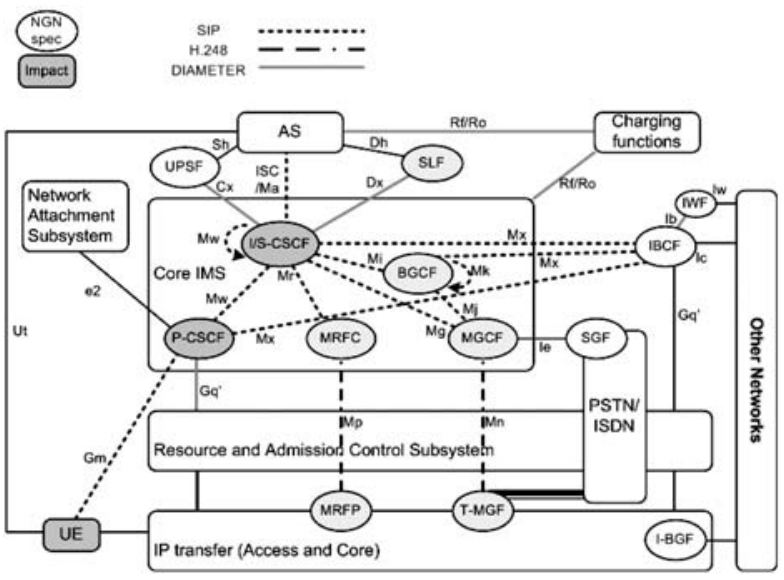

Fig. 2 Core IMS in the TISPAN_NGN

The core IMS functions [12] (Fig. 2) are included in the Call Session Control Function (CSCF) which is a SIP server which processes the IMS signalling traffic in order to control multimedia sessions. There are three types of CSCF:

- Proxy CSCF (P-CSCF): The initial point of contact for signalling traffic in to the IMS. A user is allocated a P-CSCF as a part of the registration process, and provides a two-way IPsec association with the user; all signalling traffic traverses the P-CSCF for the duration of the session. 
- Serving CSCF (S-CSCF): Provides the service coordination logic to invoke and orchestrate the application servers needed to deliver the requested service. The S-CSCF interacts with the HSS in order to determine user service eligibility by downloading the user profile; the S-CSCF is allocated for the duration of the registration.

- Interrogating CSCF (I-CSCF): A SIP proxy that provides a gateway to other domains, such as other service provider networks. TheI-CSCF may encrypt sensitive domain information a function referred to as Topology Hiding Internetwork Gateway (THIG) before forwarding the traffic.

Until Release 6, specifications for the P-CSCF included the Policy Decision Function (PDF), which stores policies and consults them to make decisions about IP bearer resource allocation requests. The PDF has been separated from the P-CSCF to make it more accessible to WLANs and other access network types. $\mathrm{P}$ CSCFs also generate CDR (Call Detail Record) or billing records that can be consolidated at a Charging Gateway Function (CGF).

Common components are those that can be accessed by more than one subsystem. Two types of common components can be identified:

- Components known in 3GPP IMS

- New components defined by TISPAN.

The first group of components has been defined by $3 \mathrm{GPP}$ IMS. It includes the following functions:

- Subscription Location Function (SLF) is only needed when multiple HSSs (Home Subscriber Servers) are used. Within TISPAN, it can be accessed by service control subsystems and Application Server Functions to retrieve the identity of the UPSF (User Profile Server Function) containing the service-level user profile of a particular subscriber.

- Application Server Function (ASF) offers value added services and resides either in the user's home network or in a third party location. The third party could be a network or simply a standalone AS.

- Interconnection Border Control Function (IBCF) which provides the interconnection with other multimedia sub-systems.

- User Profile Server Function (UPSF) which is, in fact, a subset of the HSS defined by $3 \mathrm{GPP}$. It stores all relevant information regarding the user, including identification, addressing, numbering, access controls and location information. Unlike HSS, UPSF does not provide HLR/AuC (Home Location Register/Authentication Centre) functionality.

- Charging and Data Collection Functions: As the names suggest, these provide data collection and billing mediation for online and offline charging.

The second group represents either new components that have been defined by TISPAN, or those 3GPP ones that have been modified by TISPAN in the context of NGN:

- Application Server Function (ASF) may provide standalone services or value added services on top of a basic session. For resource control purposes in NGN, the first category of Application Server Functions (ASF Type 1) may interact with the
RACS, while the second category (ASF Type 2) relies on the control subsystem that provide the basic session over which the valued added service is built. Examples of Application Server Functions are SIP Application Servers and OSA (Open Service Architecture) Application Servers. When sitting on top of the IMS, the second type of ASF (Application Server Function) is identical to the Application Server (AS) function defined by 3GPP, although a network node implementing this functional entity in an NGN network and a network node implementing it in a $3 \mathrm{GPP}$ network may differ in terms of supported services.

- Inter-working Function (IWF) is a new component that performs the inter-working between protocols used within TISPAN NGN service control subsystems and other IP-based protocols (e.g. between the SIP profile used in the IMS and other SIP profiles or IP-based protocols such as the H.323 protocol).

- Charging and Data Collection Functions include data collection functions and mediation functions to the billing systems (for supporting both on-line and off-line charging) or other management applications that may use the same data. It should be noted that charging in TISPAN Release 1 is limited to offline charging only.

\section{TISPAN NGN Releases}

NGN Release 1, published in December, 2005, provides the first set of implement-able NGN specifications that are being used by industry to build the NGN. It defines the overall architecture including IMS re-use and other subsystems.

NGN Release 2 is being completed. It builds upon Release 1 and adds in some initial applications such as Home gateway, IPTV and corporate networks. Release 2 architecture issues include new functionalities such as evolution of RACS including resource control in the core network and end-to-end QoS (Quality of Service) as well as evolution of NASS including additional access technologies, e.g., fixed access via xDSL (e.g. ADSL (Asymmetric Digital Subscriber Line), WLAN (Wireless Local Area Network) via XDSL (Digital Subscriber Line), WiMAX (Worldwide Interoperability for Microwave Access).

NGN Release 3 has been launched recently. As NGN networks are being rolled out and tested TISPAN is seeing a large increase in the number of corrections (CRs - Change Requests) to NGN Release 1 and Release 2. Some early requirements for the Release 3 include VoIP (Voice over IP) consolidation (including QoS, security, interworking), IPTV evolution, Ultra Broadband (fixed and wireless) access to the NGN and the interconnection (both IMS and non IMS).

\section{Conclusion}

The convergence between public switched telephone networks and IP based data networks forms a major part of the TISPAN work, along with the planning for the re-use in the fixed domain of the, originally mobile only, "IP Multimedia Subsystem" (IMS) developed in 3GPP. 
IMS is the coming standards-based NGN architecture of choice for today's wireless, wireline and broadband operators. The primary driver behind IMS is a move away from circuit-switched services toward IP packet-based services. However, IMS will support both systems. And because the framework is access agnostic, IMS allows operators to continue to use different underlying network architectures.

IMS is expected to respond to and solve many of the industry's biggest technological challenges, including the lack of inter- operability among operators who offer the same services and the inability of operators to take advantage of converged networks. However, IMS implementation should be viewed as a strategy of migration rather than replacement of an entire network. Operators can decide and choose which elements to implement, so the migration to IMS can be gradual. In other words, IMS can be viewed as a roadmap for operators that can guide them through an evolution to fully converged networks, services and technologies - a process that is already underway.

\section{References}

[1] http://portal.etsi.org/Portal_Common/home.asp

[2] http://www.ietf.org

[3] http://www.3gpp.org

[4] http://www.3gpp2.org

[5] http://www.ansi.org

[6] http://www.cablelabs.com

[7] http://www.msforum.org

[8] http://www.openmobilealliance.org

[9] ETSI ES 282 001: NGN Functional Architecture.

[10] ETSI ES 187 004: NGN Functional Architecture; Network Attachment Sub System (NASS).

[11] ETSI ES 187 003: Resources and Admission Control Sub-system (RACS); Functional Architecture.

[12] ETSI ES 282 007: TISPAN, IP Multimedia Subsystem (IMS): Functional Architecture. 